\title{
Espécies de Culicoides (Diptera; Ceratopogonidae) e hospedeiros potenciais em área de ecoturismo do Parque Nacional dos Lençóis Maranhenses, Brasil
}

Culicoides species (Diptera; Ceratopogonidae) and potential hosts in ecotourism area of Lençóis Maranhenses National Park, Brazil

Especies de Culicoides (Díptera; Ceratopogonidae) y huéspedes potenciales en área de ecoturismo del Parque Nacional de Lençóis Maranhenses, Brasil

Jatyna Castro Costa

Laboratório de Entomologia e Vetores, Departamento de Patologia,

Universidade Federal do Maranhão, São Luís, Maranhão, Brasil

Elias Seixas Lorosa

Laboratório Nacional e Internacional de Referência em Taxonomia de Triatomíneos, Instituto Oswaldo Cruz, Fundação Oswaldo Cruz, Rio de Janeiro, Rio de Janeiro, Brasil
Jorge Luís Pinto Moraes

Laboratório de Entomologia e Vetores, Departamento de Patologia,

Universidade Federal do Maranhão, São Luís, Maranhão, Brasil

José Manuel Macário Rebêlo

Laboratório de Entomologia e Vetores, Departamento de Patologia,

Universidade Federal do Maranhão, São Luís, Maranhão, Brasil

\section{RESUMO}

Estudou-se a riqueza, a abundância relativa e a fonte alimentar sanguínea das espécies de Culicoides em áreas rurais e urbanas do polo turístico de Barreirinhas, Estado do Maranhão, Brasil, na região do Parque Nacional dos Lençóis Maranhenses. Os insetos foram capturados em ambiente peridomiciliar, utilizando-se armadilhas luminosas HP instaladas a uma altura média de $1,5 \mathrm{~m}$, das $18 \mathrm{~h}$ às $6 \mathrm{~h}$, nos anos de 2004 a 2007 . O esforço de captura foi de 1.440 h. Foram capturados 7.964 indivíduos de 13 espécies. As mais abundantes foram Culicoides filariferus (30,7\%), C. ignacioi $(24,6 \%)$, C. insignis (13,8\%), C. leopoldoi (12,8\%), C. boliviensis $(9,9 \%)$, C. foxi (4\%) e C. paucienfuscatus $(3,2 \%)$. As demais representaram $1 \%$ da amostragem. Os Culicoides sugaram sangue de mamíferos $(54,3 \%$, incluindo humanos, $11,2 \%)$ e aves $(31,9 \%)$, sendo abundantes na zona rural, onde foram capturados, em média, 878,9 espécimes/ localidade, contra 18 espécimes/localidade na zona urbana. A elevada riqueza e abundância das espécies de Culicoides e a utilização de seres humanos e animais domésticos como hospedeiros sanguíneos indicam a necessidade de estudos entomológicos futuros para saber se o turismo, uma das principais atividades econômicas locais, vem afetando os habitats e provocando alterações na estrutura das comunidades desses insetos.

Palavras-chave: Maruins; Insetos Vetores; Distribuição Espacial da População; Hábitos Alimentares.

\section{INTRODUÇÃO}

A família Ceratopogonidae (Diptera, Nematocera) compreende um táxon de insetos de pequenas dimensões, que variam entre 1-6 mm de comprimento, conhecidos popularmente como maruins, mosquitosdo-mangue e mosquitos-pólvora'. Este grupo é bastante diversificado, contendo cerca de 5.978 espécies descritas no mundo ${ }^{2}$.

Dentre as cinco subfamílias conhecidas, Ceratopogoninae é uma das mais estudadas, tendo

\footnotetext{
Correspondência / Correspondence / Correspondencia:

José Manuel Macário Rebêlo

Universidade Federal do Maranhão

Departamento de Patologia

Praça Madre Deus, 02

CEP: 65025-560 São Luís-Maranhão-Brasil

E-mail:macariorebelo@uol.com.br
}

116 gêneros catalogados no mundo. Destes, destacase o gênero Culicoides Latreille, 1809, um grupo cosmopolita (ausente apenas na Antártica e na Nova Zelândia) que contém 1.322 espécies formalmente descritas distribuídas no mundo, das quais pelo menos 266 ocorrem na Região Neotropical ${ }^{2,3}$. No Brasil são conhecidas pelo menos 103 espécies, 4, das quais 82 ocorrem na Amazônia brasileira ${ }^{1,3,5,6,7}$, sendo que a maioria dos levantamentos entomológicos voltados para o conhecimento da diversidade desse gênero foi feita nos Estados do Amazonas $8,9,10,11,12$ e Pará ${ }^{13,14,15,16}$. Em áreas extra-amazônicas do Brasil, estudos dessa natureza ainda são poucos, tendo-se conhecimento dos inquéritos entomológicos realizados nos estados de Minas Gerais ${ }^{17}$ e Rio de Janeiro, onde já foi registrada a ocorrência de pelo menos 25 espécies $^{18}$. No Maranhão, onde os estudos sobre ceratopogonídeos são ainda incipientes, Silva e Rebêlo ${ }^{19}$ relataram a ocorrência de 15 espécies presentes na ilha de São Luís. 
As espécies deste gênero são encontradas desde o nível do mar até $4.000 \mathrm{~m}$ de altitude, alimentando-se $96 \%$ delas do sangue de mamíferos e aves ${ }^{20}$, podendo transmitir patógenos entre os vertebrados dos quais sugam sangue.

Ao praticarem a hematofagia, os maruins podem funcionar como vetores de várias espécies de parasitas. Assim, nas áreas neotropicais há relato de que filárias podem ser transmitidas por maruins ${ }^{21}$, como sucede com Mansonella ozzardi (Manson, 1897) que no norte da Argentina é transmitida para humanos pelo Culicoides lahillei (lches, 1906) ${ }^{22}$ e, no Brasil, com Onchocerca cervicallis (Railliet \& Henry, 1910), que suspeita-se seja transmitida por Culicoides spp. ${ }^{23}$. Do mesmo modo, C. paraensis (Goeldi, 1905) transmite o vírus do Oropouche $24,25,26$, C. insignis Lutz, 1913 e C. pusillus (Lutz, 1955) são potenciais vetores do vírus da Língua Azul para animais domésticos e ruminantes silvestres $27,28,29,30,31,32$. Há relato também da transmissão de protozoários ${ }^{2}$.

Além disso, as picadas de fêmeas de maruins podem causar desconforto, insônia, irritabilidade e reações alérgicas em seus hospedeiros. Nesse sentido, o ataque que algumas espécies de Culicoides spp. realizam sobre vertebrados, incluindo o homem, pode causar impacto no desenvolvimento de áreas úteis para a agricultura, pecuária e turismo, tornando esse gênero de grande importância não apenas para a medicina humana e veterinária, mas também para a economia e o meio ambiente.

Contudo, existem várias áreas potenciais para a ocorrência de Culicoides ${ }^{19,33}$. Dentre elas, destaca-se a região do Parque Nacional dos Lençóis Maranhenses (PNLM), um importante polo turístico do Estado, onde casos de doenças dermatológicas têm sido notificados $^{34,35}$. A realização de um inquérito entomológico sistematizado nessa região poderia fornecer importantes subsídios para análise futura do impacto que os maruins podem vir a causar nas áreas destinadas ao turismo e, ao mesmo tempo, a influência desta atividade econômica sobre a composição das espécies desses insetos.

Nesse sentido, realizou-se este trabalho com o objetivo de determinar a riqueza, a abundância relativa, a distribuição espacial e as fontes alimentares sanguíneas das espécies de Culicoides em localidades situadas nas rotas utilizadas pelas atividades do turismo.

\section{MATERIAIS E MÉTODOS}

\section{ÁREA DE ESTUDO}

$\bigcirc$ presente estudo foi realizado no Município de Barreirinhas $\left(2^{\circ} 45^{\prime} \mathrm{S}\right.$ e $\left.42^{\circ} 5^{\prime} \mathrm{W}\right)$, o qual possui uma área de 3.111 km² e situa-se a 266 km de São Luís, capital do Estado do Maranhão. A população é de 43 mil habitantes distribuídos principalmente na zona rural (67\%), concentrando-se o restante (33\%) na sede municipal ${ }^{36}$. Limita-se com os Municípios de Urbano Santos e Santa Quitéria (ao sul), São Bernardo (sudeste), Tutoia e Paulino Neves (nordeste), Primeira Cruz (sudoeste) e Santo Amaro (oeste).
Município situa-se na zona climática semiúmida que cruza o Estado do Maranhão transversalmente, de norte a sul. A precipitação pluviométrica média é de $1.900 \mathrm{~mm}$ anuais, sendo que $96 \%$ das chuvas se precipitam nos meses de dezembro a junho (estação chuvosa) e 4\%, de julho a novembro (estação seca).

A área de estudo propriamente dita foi composta por 12 localidades, das quais nove eram rurais (Baixa D'Água, Jabuti, Mutuns, Olho D'Água, Piquizeiro, Sobradinho e Varas - estas situadas às margens do rio Preguiças, o principal curso d'água do Município; Palmeira dos Reis, à margem do rio da Formiga; e Cocal, à margem do rio de mesmo nome); e três urbanas (Cidade Nova, Residencial Brasil e Santarém). Como todas as localidades estudadas estavam à margem do rio Preguiças ou de seus afluentes, a vegetação dominante foi a mata ciliar, situada dentro do domínio da restinga, o principal ecossistema da região de entorno do PNLM.

\section{AMOSTRAGEM}

As capturas dos maruins foram feitas na estação seca em Olho D'Água (agosto/2004), Cocal e Palmeira dos Reis (julho/2005); e na chuvosa em Baixa D'Água, Jabuti, Mutuns, Piquizeiro, Sobradinho e Varas (janeiro/2005), Cidade Nova, Residencial Brasil e Santarém (junho/2007). Foram utilizadas armadilhas luminosas HP (Hoover Pugedo) tipo CDC, mantidas por quatro pilhas grandes de 1,5 volts. Em cada localidade foram selecionadas dez casas e, em cada uma, foi instalada uma armadilha a uma altura média de 1,5 m acima do solo, em abrigo de animais domésticos (galinheiro, chiqueiro ou estábulo). Cada uma funcionou $12 \mathrm{~h}$ ininterruptas (18 h às $6 \mathrm{~h}$ ). 0 esforço de captura foi de dez armadilhas $\times 12 \mathrm{~h} \times 12$ localidades, totalizando 1.440 h. Os insetos retidos nas armadilhas foram transportados para o Laboratório de Entomologia e Vetores (LEV), do Departamento de Patologia da Universidade Federal do Maranhão, onde foram separados de outros insetos e identificados pelos técnicos e pesquisadores do LEV sob estereomicroscópio Zeiss Stemi SV6 (10 a 50X) e microscópio ótico Zeiss Primo Star (10 a 100X), ambos com luz branca, auxiliados pelas propostas de Wirth e Blanton', Wirth et $a^{37}$ e Spinelli et $a^{38}$. Uma parte dos espécimes foi fixada em álcool a $70 \%$ e incorporada à coleção entomológica do LEV. A outra parte, constituída por fêmeas ingurgitadas foi separada para a realização do teste de precipitina a fim de determinar a fonte alimentar sanguínea, cuja técnica e procedimento estão descritos em Dias et al $^{39}$.

\section{ANÁLISE ESTATÍSTICA}

ranque de abundância das espécies de Culicoides foi determinado pelo índice de Kato et $\mathrm{al}^{40}$, sendo dominantes aquelas cujo limite de confiança inferior era maior que o limite superior para espécies ausentes. A riqueza de espécies em cada localidade foi estimada pela soma do número de espécies obtidas. O índice de Shannon-Wiener foi usado para avaliar a diversidade ${ }^{41}$, sendo estimada pelo procedimento de 
Jackknife ${ }^{42}$. $O$ índice de Pielou foi usado para avaliar a equitabilidade.

\section{RESULTADOS}

\section{RIQUEZA E ABUNDÂNCIA RELATIVA DAS ESPÉCIES}

No total foram capturados 7.964 exemplares de 13 espécies de Culicoides, a saber: C. aitkeni Wirth \& Blanton, 1968; C. boliviensis Spinelli \& Wirth, 1984; C. fernandoi Tavares \& Souza, 1978; C. foxi Ortiz, 1950; C. guyanensis Floch \& Abonnenc, 1942; C. ignacioi Forattini, 1957; C. insignis Lutz, 1913; C. leopoldoi Ortiz, 1951; C. paraensis (Goeldi 1905); C. paucienfuscatus Barbosa, 1947; C. filariferus Hoffman, 1939; C. travassosi Forattini, 1957 e Culicoides sp.
As espécies mais abundantes corresponderam a 99\% da amostra total, a saber: C. filariferus $(30,7 \%)$, C. ignacioi $(24,6 \%)$, C. insignis $(13,8 \%), C$. leopoldoi $(12,8 \%)$, C. boliviensis (9,9\%), C. foxi (4\%) e C. paucienfuscatus $(3,2 \%)$ (Tabela 1).

A riqueza de espécies foi maior em Olho D'Água e Baixa D'Água (dez espécies, cada uma), Piquizeiro, Cocal e Jabuti (oito espécies) e Mutuns (sete espécies). Contudo, a abundância de indivíduos foi maior no Cocal $(37,2 \%)$, Baixa D'Água (17,7\%), Piquizeiro (17\%) e Olho D'Água (15\%), totalizando $86,9 \%$ da amostra total. Nessas localidades se destacaram, respectivamente, as espécies: C. ignacioi, C. leopoldoi, C. filarifererus e C. insignis (Tabela 1).

Tabela 1 - Espécies e respectivos números de espécimes de Culicoides coletados no Município de Barreirinhas, Estado do Maranhão, Brasil, nos anos de 2004 a 2007

\begin{tabular}{|c|c|c|c|c|c|c|c|c|c|c|c|c|c|c|}
\hline Ambientes & & & & & Rurc & & & & & & Irbanas & & Tot & tal \\
\hline Espécies / Localidades & 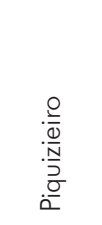 & $\begin{array}{l}\bar{O} \\
\dot{O}\end{array}$ & 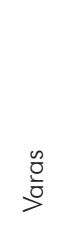 & 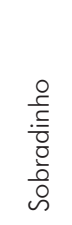 & 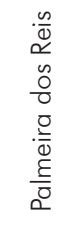 & 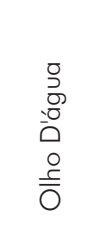 & $\begin{array}{l}\overline{\overline{0}} \\
\text { 음 }\end{array}$ & 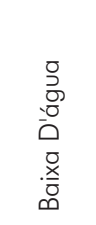 & $\begin{array}{l}\frac{n}{5} \\
\frac{2}{5} \\
\sum\end{array}$ & 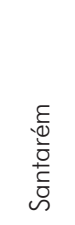 & 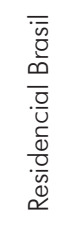 & $\begin{array}{l}0 \\
0 \\
Z \\
0 \\
0 \\
\frac{0}{0} \\
\dot{0} U\end{array}$ & No & $\%$ \\
\hline $\begin{array}{l}\text { C. filariferus Hoffman, } \\
1939\end{array}$ & 585 & 1156 & - & - & - & 385 & 18 & 264 & 41 & - & - & - & 2.449 & 30,7 \\
\hline $\begin{array}{l}\text { C. ignacioi Forattini, } \\
1957\end{array}$ & 139 & 1470 & - & - & 1 & 8 & 14 & 314 & 12 & 2 & - & - & 1.960 & 24,6 \\
\hline C. insignis Lutz, 1913 & 71 & 130 & - & - & 6 & 597 & 171 & 89 & 17 & 14 & - & 1 & 1.096 & 13,8 \\
\hline $\begin{array}{l}\text { C. leopoldoi Ortiz, } \\
1951\end{array}$ & 66 & 24 & 2 & 22 & 1 & 16 & 130 & 352 & 371 & 2 & 27 & 4 & 1.017 & 12,8 \\
\hline $\begin{array}{l}\text { C. boliviensis Spinelli } \\
\text { \& Wirth, } 1984\end{array}$ & 187 & 88 & 3 & 3 & 4 & 138 & 40 & 305 & 21 & 1 & - & 2 & 792 & 9,9 \\
\hline C. foxi Ortiz, 1950 & 78 & 67 & - & - & 18 & 36 & 5 & 63 & 53 & 1 & - & - & 321 & 4,0 \\
\hline $\begin{array}{l}\text { C. paucienfuscatus } \\
\text { Barbosa, } 1947\end{array}$ & 228 & - & - & - & - & 1 & 2 & 12 & 9 & - & - & - & 252 & 3,2 \\
\hline $\begin{array}{l}\text { C. travassosi Forattini, } \\
1957\end{array}$ & - & 28 & - & - & - & - & - & - & - & - & - & - & 28 & 0,3 \\
\hline $\begin{array}{l}\text { C. guyanensis Floch \& } \\
\text { Abonnenc, } 1942\end{array}$ & - & - & - & 21 & - & - & - & - & - & - & - & - & 21 & 0,3 \\
\hline Culicoides sp. & 4 & - & - & - & - & 2 & 1 & 6 & - & - & - & - & 13 & 0,2 \\
\hline $\begin{array}{l}\text { C. aitkeni Wirth \& } \\
\text { Blanton, } 1968\end{array}$ & - & - & - & - & - & 8 & - & 1 & - & - & - & - & 9 & 0,1 \\
\hline $\begin{array}{l}\text { C. fernandoi Tavares \& } \\
\text { Sousa, } 1978\end{array}$ & - & 3 & - & - & - & 2 & - & - & - & - & - & - & 5 & 0,1 \\
\hline $\begin{array}{l}\text { C. paraensis (Goeldi, } \\
\text { 1905) }\end{array}$ & - & - & - & - & - & - & - & 1 & - & - & - & - & 1 & - \\
\hline Total de indivíduos & 1.358 & 2.966 & 5 & 46 & 30 & 1.193 & 381 & 1.407 & 524 & 20 & 27 & 7 & 7.964 & 100,0 \\
\hline Números percentuais & 17,0 & 37,2 & 0,1 & 0,6 & 0,4 & 15,0 & 4,8 & 17,7 & 6,6 & 0,2 & 0,3 & 0,1 & 100,0 & \\
\hline Total de espécies & 8 & 8 & 2 & 3 & 5 & 10 & 8 & 10 & 7 & 5 & 1 & 3 & & \\
\hline
\end{tabular}

Sinal convencional utilizado: - Dado numérico igual a zero não resultante de arredondamento. 
O teste de Shannon revelou que Baixa D'Água foi a localidade com maior índice de diversidade $\left(H^{\prime}=1,715\right)$ seguida de Piquizeiro $\left(H^{\prime}=1,651\right)$, Jabuti $\left(H^{\prime}=1,329\right)$ e Olho D'Água $\left(H^{\prime}=1,219\right)$. Varas foi a localidade com menor índice de diversidade $\left(H^{\prime}=0,673\right)$, uma vez que Residencial Brasil só apresentou uma espécie. Quanto à equitabilidade, Varas atingiu o maior índice $(E=0,971)$, seguida por Cidade Nova $(E=0,8699)$ e Sobradinho $(0,809)$ (Tabela 2).

\section{DISTRIBUIÇÃO ESPACIAL}

Seis espécies estão distribuídas em pelo menos 50\% das localidades: C. leopoldoi (100\%), C. boliviensis $(91,6 \%)$, C. insignis (75\%), C. ignacioi, C. foxi $(66,6 \%)$ e C. filariferus (50\%); as demais ocorreram em menos da metade das localidades: C. paucienfuscatus $(41,6 \%)$, Culicoides sp. (33,3\%), C. aitkeni, C. fernandoi $(16,6 \%)$, C. travassosi, C. guyanensis e C. paraensis $(8,3 \%)$.

Tabela 2 - Índices de diversidade e equitabilidade de espécies de Culicoides nas localidades amostradas no Município de Barreirinhas, Estado do Maranhão, Brasil, nos anos de 2004 a 2007

\begin{tabular}{lccc}
\hline & & Índices & \\
\cline { 2 - 4 } & Localidades & Exponencial & Equitabilidade \\
\hline Baixa D'Água & 1,715 & 5,528961 & 0,7447 \\
Piquizeiro & 1,651 & 5,20698 & 0,7941 \\
Jabuti & 1,329 & 3,743421 & 0,639 \\
Olho D'Água & 1,219 & 3,353485 & 0,5294 \\
Cocal & 1,132 & 3,095657 & 0,5445 \\
Palmeira dos Reis & 1,124 & 3,064854 & 0,6982 \\
Mutuns & 1,072 & 2,915379 & 0,5509 \\
Santarém & 1,01 & 2,745601 & 0,6274 \\
Cidade Nova & 0,9557 & 2,58571 & 0,8699 \\
Sobradinho & 0,8888 & 2,4109 & 0,809 \\
Varas & 0,673 & 1,954237 & 0,971 \\
\hline
\end{tabular}

\section{FONTE ALIMENTAR SANGUÍNEA}

Dentre os exemplares capturados, 354 estavam ingurgitados e foram selecionados para a realização do teste de precipitina. As reações simples somaram 304, ou $85,88 \%$ das análises (Tabela 3). Os outros 50 espécimes estavam ingurgitados com sangue de dois tipos de animais, resultando, portanto, em reações duplas com diferentes combinações (Tabela 4).

Com relação às reações simples, os mamíferos foram os animais mais procurados (54,3\% da amostra total), seguidos pelas aves $(31,9 \%)$ (Tabela 3). Os mamíferos mais procurados foram cavalo $(13,8 \%)$, roedor $(12,5 \%)$ e humano $(11,2 \%)$, sendo que $13,8 \%$ das amostras não reagiram a nenhum antissoro utilizado. As espécies que sugaram maior número de espécies animais foram $C$. boliviensis, C. insignis, C. leopodoi e C. filariferus, tendo sugado todos os animais reagentes, além de apresentarem amostras não reagentes.

Foram detectados 15 tipos de reações duplas, com o predomínio de ave/boi, boi/cavalo e ave/cavalo com 14\%, cada uma (Tabela 4). Os culicoides que apresentaram maior variedade de reações duplas foram C. leopoldoi (nove reações), C. filariferus (sete) e C. ignacioi (seis). Os hospedeiros sanguíneos mais frequentes nas reações duplas foram as aves (seis reações).

Tabela 3 - Números absolutos e percentuais de reações simples do teste de precipitina em fêmeas de Culicoides ingurgitadas, no Município de Barreirinhas, Estado do Maranhão, Brasil, nos anos de 2004 a 2007

\begin{tabular}{|c|c|c|c|c|c|c|c|c|c|c|c|c|c|c|c|c|}
\hline \multirow{2}{*}{ Vertebrados } & \multicolumn{2}{|c|}{ C. aikeni } & \multicolumn{2}{|c|}{ C. boliviensis } & \multicolumn{2}{|c|}{ C. foxi } & \multicolumn{2}{|c|}{ C. ignacioi } & \multicolumn{2}{|c|}{ C. insignis } & \multicolumn{2}{|c|}{ C. leopodoi } & \multicolumn{2}{|c|}{$\begin{array}{l}\text { C. pseudo- } \\
\text { diabolicus }\end{array}$} & \multicolumn{2}{|c|}{ Total } \\
\hline & N & $\%$ & $\mathrm{~N}$ & $\%$ & $\mathrm{~N}$ & $\%$ & $\mathrm{~N}$ & $\%$ & $\mathrm{~N}$ & $\%$ & $\mathrm{~N}$ & $\%$ & $N$ & $\%$ & $\mathrm{~N}$ & $\%$ \\
\hline Ave & 4 & 66,8 & 24 & 32,9 & 7 & 38,9 & 16 & 31,4 & 12 & 38,7 & 18 & 28,6 & 16 & 25,8 & 97 & 31,9 \\
\hline Cavalo & 1 & 16,6 & 13 & 17,8 & 2 & 11,1 & 10 & 19,6 & 4 & 13,0 & 4 & 6,4 & 8 & 12,9 & 42 & 13,8 \\
\hline Roedor & - & - & 8 & 11,0 & 3 & 16,8 & 6 & 11,8 & 2 & 6,4 & 13 & 20,6 & 6 & 9,7 & 38 & 12,5 \\
\hline Gambá & - & - & 3 & 4,1 & 2 & 11,1 & - & - & 2 & 6,4 & 6 & 9,5 & 4 & 6,5 & 17 & 5,6 \\
\hline Humano & 1 & 16,6 & 6 & 8,2 & 2 & 11,1 & 6 & 11,8 & 2 & 6,4 & 6 & 9,5 & 11 & 17,7 & 34 & 11,2 \\
\hline Cão & - & - & 5 & 6,7 & - & - & 5 & 9,8 & 2 & 6,4 & 4 & 6,4 & 2 & 3,2 & 18 & 5,9 \\
\hline Boi & - & - & 6 & 8,2 & 1 & 5,5 & 3 & 5,8 & 1 & 3,3 & 2 & 3,1 & 3 & 4,8 & 16 & 5,3 \\
\hline $\begin{array}{l}\text { Não } \\
\text { reagentes }\end{array}$ & - & - & 8 & 11,0 & 1 & 5,5 & 5 & 9,8 & 6 & 19,4 & 10 & 15,9 & 12 & 19,4 & 42 & 13,8 \\
\hline Total & 6 & 2,0 & 73 & 24,0 & 18 & 5,9 & 51 & 16,8 & 31 & 10,2 & 63 & 20,7 & 62 & 20,4 & 304 & 100,0 \\
\hline
\end{tabular}

Sinal convencional utilizado: - Dado numérico igual a zero não resultante de arredondamento. 
Tabela 4 - Números absolutos e percentuais de reações duplas do teste de precipitina em fêmeas de Culicoides ingurgitadas, no Município de Barreirinhas, Estado do Maranhão, Brasil, nos anos de 2004 a 2007

\begin{tabular}{|c|c|c|c|c|c|c|c|c|c|c|c|c|c|c|c|c|}
\hline \multirow{3}{*}{ Vertebrados } & \multicolumn{14}{|c|}{ Espécies } & \multirow{2}{*}{\multicolumn{2}{|c|}{ Total }} \\
\hline & \multicolumn{2}{|c|}{ C. aikeni } & \multicolumn{2}{|c|}{ C. boliviensis } & \multicolumn{2}{|c|}{ C. foxi } & \multicolumn{2}{|c|}{ C. ignacioi } & \multicolumn{2}{|c|}{ C. insignis } & \multicolumn{2}{|c|}{ C. leopodoi } & \multicolumn{2}{|c|}{$\begin{array}{l}\text { C. pseudo- } \\
\text { diabolicus }\end{array}$} & & \\
\hline & $\mathrm{N}$ & $\%$ & N & $\%$ & $\mathrm{~N}$ & $\%$ & $\mathrm{~N}$ & $\%$ & $\mathrm{~N}$ & $\%$ & $\mathrm{~N}$ & $\%$ & $\mathrm{~N}$ & $\%$ & $\mathrm{~N}$ & $\%$ \\
\hline Ave/boi & 1 & 33,3 & - & - & - & - & 3 & 30 & - & - & 1 & 8,3 & 2 & 16,6 & 7 & 14,0 \\
\hline Cavalo/roedor & 1 & 33,3 & - & - & - & - & - & - & - & - & - & - & - & - & 1 & 2,0 \\
\hline Ave/cão & 1 & 33,3 & - & - & - & - & 1 & 10 & 2 & 40 & 1 & 8,3 & - & - & 5 & 10,0 \\
\hline Gambá/humano & - & - & 1 & 20 & - & - & - & - & - & - & 1 & 8,3 & - & - & 2 & 4,0 \\
\hline Ave/roedor & - & - & 1 & 20 & - & - & 1 & 10 & - & - & - & - & 1 & 8,3 & 3 & 6,0 \\
\hline Cão/cavalo & - & - & 2 & 40 & 1 & 33,3 & - & - & - & - & - & - & - & - & 3 & 6,0 \\
\hline Ave/humano & - & - & 1 & 20 & - & - & - & - & - & - & - & - & 2 & 16,6 & 3 & 6,0 \\
\hline Boi/cavalo & - & - & - & - & 1 & 33,3 & 2 & 20 & - & - & 1 & 8,3 & 3 & 25 & 7 & 14,0 \\
\hline Humano/roedor & - & - & - & - & 1 & 33,3 & 1 & 10 & - & - & - & - & - & - & 2 & 4,0 \\
\hline Ave/cavalo & - & - & - & - & - & & 2 & 20 & 1 & 20 & 2 & 16,7 & 2 & 16,6 & 7 & 14,0 \\
\hline Ave/gambá & - & - & - & - & - & - & - & - & 1 & 20 & 2 & 16,7 & - & - & 3 & 6,0 \\
\hline Cão/humano & - & - & - & - & - & - & - & - & 1 & 20 & - & - & 1 & 8,3 & 2 & 4,0 \\
\hline Gambá/roedor & - & - & - & - & - & - & - & - & - & - & 1 & 8,3 & 1 & 8,3 & 2 & 4,0 \\
\hline Cavalo/gambá & - & - & - & - & - & - & - & - & - & - & 2 & 16,7 & - & - & 2 & 4,0 \\
\hline Cão/gambá & - & - & - & - & - & - & - & - & - & - & 1 & 8,3 & - & - & 1 & 2,0 \\
\hline Total & 3 & 6,0 & 5 & 10,0 & 3 & 6,0 & 10 & 20,0 & 5 & 10,0 & 12 & 24,0 & 12 & 24,0 & 50 & 100,0 \\
\hline
\end{tabular}

Sinal convencional utilizado: - Dado numérico igual a zero não resultante de arredondamento.

\section{DISCUSSÃO}

A fauna de Culicoides da região Parque Nacional dos Lençóis Maranhenses mostrou-se diversificada, sendo representada por seis espécies que ainda não haviam sido notificadas para o Estado do Maranhão: C. aitkeni, C. boliviensis, C. fernandoi, C. filarifer e C. travassosi e uma não determinada, além de sete espécies registradas também na ilha de São Luís (C. foxi, C. guyanensis, C. ignacioi, C. insignis, C. leopoldoi, C. paraensis e C. paucienfuscatus) ${ }^{19}$.

Neste estudo, ainda que as coletas tenham sido feitas em uma única noite nas localidades selecionadas, o número de espécies encontradas (13) foi relativamente alto quando se observa a quantidade de espécies obtidas nos estudos levados a efeito, com diferentes métodos, em outros Estados da Amazônia brasileira. No Estado do Amazonas, por exemplo, foram encontrados números variáveis de seis a 23 espécies nos diversos inquéritos entomológicos realizados $8,9,10,11,12$, enquanto no vizinho Estado do Pará, os estudos entomológicos notificaram a ocorrência de cinco a 18 espécies ${ }^{13,14,15,16}$.

As espécies mais abundantes neste estudo - C. filariferus, C. ignacioi, C. insignis, C. leopoldoi, C. boliviensis, C. foxi e C. paucienfuscatus - ocorreram em várias localidades e interagiram com uma variedade maior de animais que podem funcionar como fonte alimentar sanguínea. Estas características são coerentes com a valência ecológica e ampla distribuição geográfica que estas espécies apresentam?.

As outras espécies, pela baixa frequência, parecem raras quando comparadas com aquelas supracitadas e, por conseguinte, pouca antropofílicas. Porém, em inquéritos entomológicos é comum que algumas espécies apareçam em baixa frequência, reflexo do método utilizado (esforço de captura, características dos ambientes de coletas, presença de animais, etc.). Desta forma, é possível que nos locais mais distantes dos peridomicílios a abundância dessas espécies seja restabelecida.

Observou-se, neste estudo, que a riqueza e abundância de maruins foram maiores nas localidades rurais. Tal padrão de ocorrência, por um lado, se justifica pelo esforço amostral maior na zona rural. Por outro lado, observa-se a existência de condições ambientais e bioecológicas mais favoráveis nas localidades rurais, pois estas ficam próximas à vegetação natural que se desenvolve às margens dos corpos d'água (rio, riachos e córregos), onde se formam os alagadiços e brejos. Nesses ambientes úmidos, há um maior afluxo de maruins ${ }^{15,43}$, que acabam invadindo os ambientes peridomiciliares próximos, onde frequentemente se encontram aglomerados de animais domésticos, potenciais hospedeiros sanguíneos para estes insetos. É conhecida, por exemplo, a atração de C. foxi Ortiz, 1950 por mulas ou cavalos e C. insignis Lutz por gado bovino ${ }^{14}$, nesses ambientes.

Por outro lado, a modificação das matas e brejos, por meio de atividades de desmatamentos e aterros, respectivamente, pode diminuir a oferta de recursos chaves, como alimento, abrigo e criadouro nos ecótopos naturais. Esse processo pode intensificar a chegada dos maruins aos núcleos rurais, principalmente se não houver melhorias sanitárias e ambientais nos núcleos rurais. Em consequência e, por causa do hábito hematofágico, as fêmeas de maruins podem constituir verdadeiras pragas para o homem e vertebrados domésticos ${ }^{44}$.

Já naquelas áreas onde a urbanização é mais intensa, como na sede municipal de Barreirinhas, a presença dos maruins é menos frequente, indicativo de que esse 
tipo de ambiente pode constituir uma barreira para o desenvolvimento desses insetos devido, sobretudo, a redução de ecótopos favoráveis para a sua proliferação.

Para confirmar tal hipótese, estimula-se fortemente a realização de monitoramento entomológico nos arredores dos povoados rurais e na periferia urbana. $\bigcirc$ propósito é detectar se há mudanças na composição e abundância das espécies de maruins, em função das transformações ambientais decorrentes, sobretudo, do crescimento do turismo, a principal atividade econômica da região deste estudo, que vem contribuindo para a destruição e fragmentação dos habitats naturais.

Em adição, considerando que os maruins usam animais domésticos e também as pessoas como fonte sanguínea, conforme demonstrado neste estudo, torna-se necessário investigar se há relatos de agravos à saúde nas comunidades locais e em turistas, causados pelas picadas produzidas e/ou patógenos transmitidos por esses insetos.

\section{CONCLUSÃO}

Os dados apresentados mostram que várias espécies de maruins ocorrem nas diversas localidades do entorno do PNLM, com frequência variável. As espécies mais abundantes foram C. filariferus, C. ignacioi, C. insignis, C. leopoldoi e C. boliviensis. Os maruins se alimentaram de diferentes grupos de vertebrados, principalmente de sangue de cavalo, roedor, humano e ave. C. boliviensis foi a mais eclética, tendo sugado o maior número de animais e C. leopoldoi foi a mais bem distribuída, sendo encontrada em todas as localidades estudadas.

\title{
Culicoides species (Diptera; Ceratopogonidae) and potential hosts in ecotourism area of Lençóis Maranhenses National Park, Brazil
}

\begin{abstract}
Richness, relative abundance, and blood food source of Culicoides species were studied in rural and urban areas in Barreirinhas Brazilian tourist center, Maranhão State, in Lençóis Maranhenses National Park area. Insects were collected in peridomiciliary environment using HP light traps placed about $1.5 \mathrm{~m}$ above the floor, from 6:00 pm to 6:00 am, in the years 2004 to 2007. The effort was 1,440 h. A total of 7,964 individuals belonging to 13 species were caught. The most abundant ones were Culicoides filariferus (30.7\%), C. ignacioi $(24.6 \%)$, C. insignis $(13.8 \%)$, C. leopoldoi (12.8\%), C. boliviensis (9.9\%), C. foxi (4\%), and C. paucienfuscatus (3.2\%). The others were $1 \%$ of sampling. Culicoides sucked blood of mammals $(54.3 \%$, including humans, $11.2 \%)$ and birds (31.9\%), more abundant in rural area where the average of catches was 878.9 specimens per locality compared to 18 specimens per locality in urban area. The high wealth and abundance of Culicoides species, and humans and domestic animals as blood hosts, have indicated future entomological studies are necessary to determine whether the tourism, one of the main local economic activities, has affected habitats and changed the community structure of those insects.
\end{abstract}

Keywords: Culicoides; Insect Vectors; Residence Characteristics; Food Habits.

\section{Especies de Culicoides (Díptera; Ceratopogonidae) y huéspedes potenciales en área de ecoturismo del Parque Nacional de Lençóis Maranhenses, Brasil}

\section{RESUMEN}

Se estudió la riqueza, abundancia relativa y la fuente alimentar sanguínea de las especies de Culicoides en áreas rurales y urbanas del polo turístico de Barreirinhas, Estado de Maranhão, Brasil, en la región del Parque Nacional de Lençóis Maranhenses. Los insectos fueron capturados en ambiente peridomiciliario, utilizando trampas luminosas HP instaladas a una altura promedio de $1,5 \mathrm{~m}$, de las $18 \mathrm{~h}$ a las $6 \mathrm{~h}$, los años de 2004 a 2007 . El esfuerzo de captura fue de $1.440 \mathrm{~h}$. Se capturaron 7.964 individuos de 13 especies. Las más abundantes fueron Culicoides filariferus $(30,7 \%)$, C. ignacioi $(24,6 \%)$, C. insignis $(13,8 \%)$, C. leopoldoi $(12,8 \%)$, C. boliviensis $(9,9 \%)$, C. foxi (4\%) y C. paucienfuscatus $(3,2 \%)$. Las demás representaron $1 \%$ del muestreo. Los Culicoides chuparon sangre de mamíferos $(54,3 \%$, incluyendo a humanos, $11,2 \%)$ y de aves $(31,9 \%)$, siendo abundantes en la zona rural, en donde fueron capturados, en promedio, 878,9 especímenes/localidad, contra 18 especímenes/localidad en la zona urbana. La elevada riqueza y abundancia de las especies de Culicoides y la utilización de seres humanos y animales domésticos como huéspedes sanguíneos indica la necesidad de estudios entomológicos futuros para saber si el turismo, una de las principales actividades económicas locales, está afectando a los hábitats y provocando alteraciones en la estructura de las comunidades de esos insectos.

Palabras clave: Culicoides; Insectos Vectores; Distribución Espacial de la Población; Hábitos Alimenticios. 


\section{REFERÊNCIAS}

1 Wirth WW, Blanton FS. A rewiew of the maruins or biting midges of the genus Culicoides (Diptera: Ceratopogonidae) in the Amazon Basin. Amazoniana. 1973;4(4):405-70.

2 Borkent A. World species of biting midges (Diptera: Ceratopogonidae) [Internet] 2009. Salmon Arm, Canada in association with the Royal British Columbia Museum, American Museum of Natural History, and Instituto Nacional de Biodiversidad [cited 2012 Dec 8]. Available from: www.inhs.illinois. edu/research/FLYTREE/CeratopognidaeCatalog.pdf.

3 Borkent A, Spinelli GR. Neotropical Ceratopogonidae (Diptera: Insecta). In: Adis J, Arias JR, Rueda-Delgado G, Wantzen KM, editors. Aquatic Biodiversity Latin America. Pensoft Publishers; 2007. p. 101-47.

4 Aparício AAS, Castellón GE, Fonseca RFO. Distribuição de Culicoides (Diptera: Ceratopogonidae) na Amazônia Legal através de técnicas de geoprocessamento. Rev Colombiana Cienc Anim. 2011 ;3(2):283-99.

5 Felippe-Baver ML, Oliveira SJ. Lista dos exemplares tipos de Ceratopogonidae (Diptera: Nematocera) depositados na coleção entomológica do Instituto Oswaldo Cruz, Rio de Janeiro, Brasil. Mem Inst Oswaldo Cruz. 2001 nov;96(8):1 109-19.

6 Felippe-Baver ML, Veras R, Castellón EG, Moreira NA. A new Culicoides from the Amazonian region, Brazil (Diptera: Ceratopogonidae). Mem Inst Oswaldo Cruz. 2000 Jan-Feb;95(1):35-7.

7 Felippe-Baver ML, Damasceno CP, Py-Daniel V, Spinelli GR. Culicoides baniwa sp.nov. from the Brazilian Amazon Region with a synopsis of the hylas species group (Diptera: Ceratopogonidae). Mem Inst Oswaldo Cruz. 2009 Sep;104(6):851-7.

8 Castellón EG, Ferreira RLM, Silva MNT. Culicoides (Diptera: Ceratopogonidae) na Amazônia Brasileira. I. Coletas na usina hidrelétrica (UHE) de Balbina, usina hidrelétrica (UHE) Cachoeira Porteira e Cachoeira dos Espelhos (Rio Xingu). Acta Amaz. $1990 ; 20: 77-81$.

9 Castellón EG. Culicoides (Diptera: Ceratopogonidae) na Amazônia Brasileira. II. Espécies coletadas na Reserva Florestal Ducke: aspectos ecológicos e distribuição geográfica. Acta Amaz. 1990;20:83-93.

10 Castellón EG, Ferreira RLM. Culicoides Latreille (Diptera: Ceratopogonidea) da Amazônia. III. Resultados de coletas noturnas, na Reserva Florestal Ducke, Estado do Amazonas, Brasil. Bol Mus Para Emilio Goeldi. 1991;7:117-23.

11 Castellón EG, Ferreira RLM, Silva MNT. Culicoides (Diptera: Ceratopogonidae) in the Brazilian Amazon. IV. Species collected with CDC light trap in the Ducke Forest Reserve (DFR), Amazon State, Brazil. Acta Amaz. 1993;23(2-3):309-10.
12 Veras RS, Castellón EG. Culicoides Latreille. (Diptera: Ceratopogonidae) in Brazilian Amazon. V. Efficiency of traps and baits and vertical stratification in the forest reserve Adolpho Ducke. Rev Bras Zool. 1998; 15(1):145-52.

13 Trindade RS, Gorayeb IS. Maruins (Ceratopogonidae: Diptera) do estuário do Rio Pará e do litoral do Estado do Pará, Brasil. Entomol Vect. 2005;12(1): 61-74.

14 Trindade RL, Gorayeb IS. Jejenes (Diptera: Ceratopogonidae: Culicoides), luego de la estación de lluvias, en la Reserva de Desarrollo Sostenible Itatupã-Baquiá, Gurupá, Pará, Brasil. Rev Pan-Amaz Saude. 2010;1 (2):121-30.

15 Silva FDF, Okada Y, Felippe-Bauer ML. Culicoides Latreille (Diptera: Ceratopogonidae) da vila de Alter do Chão, Santarém, Pará, Brasil. Rev Pan-Amaz Saude. 2010;1 (3):69-74.

16 Santarém MCA, Confalonieri UEC, FelippeBaver ML. Diversidade de Culicoides (Diptera: Ceratopogonidae) na Floresta Nacional de Caxivanã, Melgaço, Estado do Pará, Brasil. Rev Pan-Amaz Saude. 2010;1 (4):29-33.

17 Laender JO, Ribeiro ES, Gouveia AMG, Lobato ZIP, Felippe-Baver ML. Levantamento das espécies de Culicoides Latreille (Diptera: Ceratopogonidae) encontradas nas mesorregiões norte de Minas, Jequitinhonha e Vale do Mucuri, Minas Gerais, Brasil. Entomol Vect. 2004; 1 1 (1):145-57.

18 Silva CS, Felippe-Baver ML, Almeida EHG, Figueiredo LR. Culicoides (Diptera: Ceratopogonidae) do Estado do Rio de Janeiro. I. Região norte: município de Campos dos Goytacazes. Entomol Vect. $2001 ; 8(3): 349-58$.

19 Silva FS, Rebêlo JMM. Espécies de Culicoides Latreille (Diptera: Ceratopogonidae) da ilha de São Luís, Maranhão, Brasil. Bol Mus Para Emílio Goeldi. 1999;15:169-79.

20 Mellor PS, Boorman J, Baylis M. Culicoides biting midges: their role as arbovirus vectors. Ann Rev Entomol. 2000 Jan;45:307-40.

21 Nathan MB. Transmission of the human filarial parasite Mansonella ozzardi by Culicoides phlebotomus (Williston) (Diptera: Ceratopogonidae) in coastal north Trinidad. Bull Entomol Res. 1981 Mar;71(1):97-105.

22 Shelley AJ, Coscarón S. Simuliid blackflies (Diptera: Simuliidae) and ceratopogonid midges (Diptera: Ceratopogonidae) as vectors of Mansonella ozzardi (Nematoda: Onchocercidae) in Northern Argentina. Mem Inst Oswaldo Cruz. 2001 May;96(4):451-8.

23 Marques SM, Scroferneker ML. Onchocerca cervicalis in horses from Southern Brazil. Trop Anim Health Prod. 2004 Oct;36(7):633-6. 
24 Pinheiro FP, Travassos da Rosa APA, Gomes ML, LeDuc JW, Hoch AL. Transmission of Oropouche virus from man to hamster by the midge Culicoides paraensis. Science. 1982; 215(4537):1251-53.

25 Linley JR, Hoch AL, Pinheiro FP. Biting midges (Diptera: Ceratopogonidae) and human health. J Med Entomol. 1983 Jul;20(4):347-64.

26 Degallier N, Travassos da Rosa APA, Herve JP, Vasconcelos PFC, Travassos da Rosa JFS, Sá Filho $\mathrm{GC}$, et al. Evolutionary aspects of the ecology of arboviruses in Brazilian Amazonia, South America. In: Travassos da Rosa APA, Vasconcelos PFC, Travassos da Rosa JFS, editors. An overview of arbovirology in Brazil and neighbouring countries. Belém: Instituto Evandro Chagas; 1998. p. 42-60.

27 Greiner EC, Garris GI, Rollo RT, Knausenberguer WI, Jones JE, Gibbs EPJ. Preliminary studies on the Culicoides spp. as potential vectors of bluetongue in the Caribbean Region. Prev Vet Med. 1984 Mar;2(1-4):389-99.

28 Greiner EC, Knausenberger WI, Messersmith M, Kramer WL, Gibbs EP. Culicoides spp. (Diptera: Ceratopogonidae) associated with cattle in St. Croix, Virgin Islands, and their relevance to bluetongue viruses. J Med Entomol. 1990 Nov;27(6):1071-3.

29 Homan EJ, Mo CL, Thompson LH, Barreto $\mathrm{CH}$, Oviedo MT, Gibbs EP, et al. Epidemiologic study of bluetongue viruses in Central America and the Caribbean: 1986-1988. Regional bluetongue team. Am J Vet Res. 1990 Jul;51 (7):1089-94.

30 Tanya VN, Greiner EC, Gibbs EPJ. Evaluation of Culicoides insignis (Diptera: Ceratopogonidae) as a vector of bluetongue virus. Vet Microbiology. 1992 Jul;32(1): 1 - 14.

31 Sáenz MR, Greiner EC. Culicoides aspirated from cattle in Costa Rica, Honduras, Panama and Puerto Rico, and their role as potential vectors of the bluetongue viruses. Med Vet Entomol. 1994 Jan;8(1):15-9.

32 Laender JO, Ribeiro ES, Gouveia AMG, Lobato ZIP, Felippe-Baver ML. Levantamento das espécies de Culicoides Latreille, 1809 (Diptera: Ceratopogonidae) encontradas nas mesorregiões norte de Minas, Jequitinhonha e Vale do Mucuri, Minas Gerais, Brasil. Entomol Vectores. 2004 jan-fev; 11 (1): 145-57.

33 Barros VLL, Marinho RM, Rebêlo JMM. Ocorrência de espécies de Culicoides Latreille (Diptera: Ceratopogonidae) na área metropolitana de São Luís, Maranhão, Brasil. Cad Saude Publica. 2007 jan;23(1 1):2789-90.
34 Assunção Júnior AN, Silva $O$, Moraes JLP, Nascimento FRF, Pereira YNO, Costa JML, et al. Foco emergente de leishmaniose tegumentar no entorno do Parque Nacional dos Lençóis Maranhenses, Nordeste, Brasil. Gaz Med Bahia. 2009;79(Supl.3): 103-9.

35 Rebêlo JMM, Assuncão Júnior NA, Silva $O$, Moraes JLP. Ocorrência de flebotomíneos (Diptera, Psychodidae) em focos de leishmanioses, em área de ecoturismo do entorno do Parque Nacional dos Lençóis Maranhenses, Brasil. Cad Saude Publica. 2010 jan;26(1):195-8.

36 Instituto Brasileiro de Geografia e Estatística. Censo Demográfico [Internet]. 2010 [citado 2011 set 21]. Disponível em: http://www.ibge.gov.br.

37 Wirth WW, Dyce AL, Spinelli GR. An atlas on wing photographs, with a summary of the numerical characters of the neotropical species of Culicoides (Diptera: Ceratopogonidae). Contr Am Entomol Inst. $1988 ; 25: 1-72$.

38 Spinelli GR, Ronderos MM, Díaz F, Marino PI. The bloodsucking biting midges of Argentina (Diptera: Ceratopogonidae). Mem Inst Oswaldo Cruz. 2005 Apr;100(2):137-50.

39 Dias FOP, Lorosa ES, Rebêlo JMM. Fonte alimentar sangüínea e a peridomiciliação de Lutzomyia longipalpis (Lutz \& Neiva, 1912) (Psychodidae, Phlebotominae). Cad Saude Publica. 2003 setout; 19(5): 1373-80.

40 Kato M, Matsuda T, Yamashhita Z. Associative ecology of insects found in paddy field cultivated by various planting forms. Sci Rep Tohoku Univ. 1952;19:291-301.

41 Pielou EC. Ecological diversity. New York: Wiley InterScience; 1975. $165 \mathrm{p}$

42 Zahl S. Jackknifing an index of diversity. Ecology. 1977 Jul;58(4):907-13.

43 Kettle DS. The bionomics and control of Culicoides and Leptoconops (Diptera, Ceratopogonidae = Heleidae). Ann Rev Entomol. 1962 Jan;7:401-18.

44 Kettle DS. Biology and bionomics of bloodsucking Ceratopogonids. Ann Rev Entomol. 1977 Jan; 22:33-51. 